\title{
Beam-centric Handover Decision in Dense 5G-mmWave Networks
}

\author{
Abdulkadir Kose*, Chuan Heng Foh*, Haeyoung Lee*, Mehrdad Dianati ${ }^{\dagger}$ \\ *5GIC, Institute for Communication Systems (ICS), University of Surrey, Guildford, UK \\ ${ }^{\dagger}$ Warwick Manufacturing Group, University of Warwick, Coventry, UK \\ Email: \{a.kose, c.foh, Haeyoung.Lee\}@surrey.ac.uk, M.Dianati@warwick.ac.uk
}

\begin{abstract}
In the 5G network, dense deployment and millimetre wave (mmWave) are some of the key approaches to boost network capacity. Dense deployment of $\mathbf{m m W a v e}$ small cells using narrow directional beams will escalate the cell and beam related handovers for high mobility of vehicles, which may in turn limits the performance gain promised by $5 \mathrm{G}-\mathrm{mmWave}$ based vehicle-toinfrastructure (V2I) communication. One of the research issues in mmWave handover is to minimise the handover needs by identifying long lasting connections. In this paper, we first develop an analytical model to derive the vehicle sojourn time within a beam coverage. When multiple connections offered by nearby all mmWave small cells are available when upon a handover event, we further derive the longest sojourn time among all potential connections which represents the theoretical upperbound limit of the sojourn time performance. We then design a Fuzzy Logic (FL) based distributed beam-centric handover decision algorithm to maximise vehicle sojourn time. Simulation experiments are conducted to validate our analytical model and show the performance advantage of our proposed FLbased solution when compared with commonly used approach of connecting to the strongest connection.
\end{abstract}

Index Terms-Beam Handover, mmWave Networks, 5G.

\section{INTRODUCTION}

Due to explosive growth of data traffic, millimetre wave (mmWave) has become one of the promising candidate for $5 \mathrm{G}$ systems due to inadequacy of available spectrum and high data rate support in current microwave bands. In contrast, users often suffer from high propagation loss at higher frequencies. As a remedy, directional transmission is adopted in mmWave but it incurs new challenges in mobility management. It highly depends on accurate beam alignment and connection robustness against rapid channel changes [1] [2]. Moreover, it requires new solutions to manage beam transitions which will add additional challenges in case of high mobility vehicles [3].

Frequent handover mitigation has been studied in the literature widely [4]-[7]. The authors in [4] proposed a velocityaware handover skipping scheme in two-tier cellular networks. Its aim is to lighten the overhead from frequent handovers by bypassing association with some of base stations (BS) along the user's trajectory at the expense of sacrificing higher data rates from the best signal-to-interference-plus-noise-ratio (SINR) association. As compared to the always best SINR association scheme, the proposed method achieves significant gains in average throughput at the cost of degradation in coverage probability. In [5], a handover skipping scheme is developed to reduce impact of handover delay on throughput by forcing users not to connect some strongest BSs along the trajectory. Study shows that BS skipping scheme performs better performance in long-term average rate under high mobility and high BS densities despite reduced average coverage probability. In [6], the authors mitigated the impact of frequent handovers by skipping some of the handovers by using cell topology and user's path in the target cell with cooperative decision among three BSs located on the user path. The work [7] proposed sojourn time based small cell selection in ultra dense networks (UDNs) by estimating user displacement in a Voroni cell as a cord length with known user trajectory, showing reduction of ping-pong handovers as compared to the reference signal received power (RSRP) based scheme.

Most of these studies consider small cells at microwave band hence models are derived based on omnidirectional coverage and cannot directly be applicable to directional mmWave networks. In directional mmWave networks, handover not only occurs between cells (or inter-cell), but also between beams (or inter-beam). In other words, there are more factors on the ground and different coverage shapes make handover much more complex. Moreover, dense deployment of mmWave cells and using narrow beams will escalate even further the number of inter-cell and inter-beam handovers, which can limit the performance gain promised by 5G [8]. Hence, new techniques are required to manage beam-aware handover performance. In this regard, the works presented in [1], [8], [9] analyse handover problem in mmWave networks with directional antenna configurations. In [8], authors derived an analytical model for inter-cell handover and inter-beam handover rates to investigate the impact of handover stickiness and mmWave BS density on inter-cell handover (ICH) and inter-beam handover (IBH) performance. The authors found that both ICH and IBH frequency tend to increase with BS density and user speed, and both decrease as the setting of handover stickiness increases. To reduce IBH frequency, the work presented in [9] proposed an inter-beam handover class (IBHC) which groups and synchronizes multiple beams in according to mobile user's movement route and speed so that they do not perform handover for each beam within the same group. As a result, frequent IBHs are avoided which claimed to adequately support very high mobility (i.e. $>300 \mathrm{~km} / \mathrm{h}$ ) in mmWave networks. Focusing on connection robustness, an effective beam coverage probability (EBCP) 
based handover scheme in mmWave heterogeneous network to improve connection robustness is presented in [1]. EBCP is adopted as a handover decision criteria along with hysteresis and time-to-trigger (TTT) values. It was shown that handover frequency rate is decreased compared to traditional RSRPbased scheme at the cost of throughput.

\section{A. Our Contribution}

In this paper, we consider a densely deployed mmWave network and propose a handover decision to minimise the chance of handover events when a vehicle travels across the network. We approach the problem by first answering the fundamental question where if the moving vehicle can always connect to a mmWave beam that offers the longest sojourn time, what is the throughput performance? Finding a beam offering the longest sojourn time essentially minimises the chance of handover events. We then design a distributed algorithm that utilises the instantaneous mobility information for handover decision making. The following describes the main contributions of this paper.

- We develop an analytical model which finds the theoretical upper-bound for vehicle sojourn time between two consecutive handover events in a dense mmWave network. Specifically, we formulate directly the vehicle displacement within a beam, and derive the longest vehicle displacement among all beams visible by the vehicle. The theoretical upper-bound is also used to benchmark the performance of any practical design.

- We utilise the relative location and direction of travel of a vehicle to design a Fuzzy Logic (FL) based beam-centric distributed algorithm to determine the beam among all visible beams where a vehicle can achieve the longest displacement within it.

The remainder of the paper is organized as follows. Section II describes the system model used in this paper. In Section III, we develop an analytical model to study the vehicle displacement within a beam and the longest displacement among multiple beams. We further present the FL-based beamcentric distributed algorithm for handover decision. Section IV presents analytical and simulation results. Finally, Section V summarises our key findings and shares potential issues for future research.

\section{SYSTEM MODEL}

\section{A. Network and Vehicle Mobility Model}

We consider dense deployment of $5 \mathrm{G}$ mmWave small cell networks. The mmWave BSs deployment follows 2dimensional homogeneous Poisson point process (PPP) characterized by the network density $\lambda$. We assume no involvement of macro BS in the connection as the densely deployed mmWave BSs offer complete coverage for the network. mmWave BSs have the capability to form multiple beams with directional antennas. In this work, we neglect inter-cell interference since directional mmWave networks are shown to be noise-limited rather than interference limited. We consider straight trajectory for a moving vehicle with constant velocity.
With constant velocity, the measure of vehicle sojourn time within a beam is equivalent to the vehicle displacement within a beam. We shall model vehicle displacement in our analytical derivation. In this paper, we focus on a single vehicle scenario.

\section{B. Path Loss Model}

The coverage of each BS is divided equally into $N_{s}$ sectors with angle $\theta_{s}$ representing the beam width for each beam. Each directional beam propagation is evaluated through directivity $D$ which can be calculated by [10]

$$
D=\frac{2 \pi}{\theta_{s}} .
$$

As gain $G=k \cdot D$, assuming lossless antenna, i.e. $k=1, G$ is equal to $D$. Let $G_{t}$ and $G_{r}$ be the transmitter and receiver antenna gain, respectively. For a given transmit power $P_{t}$, the received power $P_{r}$ can be calculated by

$$
P_{r}=P_{t}+G_{t}+G_{r}-P L(d),
$$

where $P L(d)$ is the path loss component, and $d$ is the distance between transmitter and receiver. $P L$, can be further determined by

$$
P L(d)=P L_{0}+10 \cdot \alpha \cdot \log (d) .
$$

Here, $P L_{0}=20 \cdot \log \left(\frac{4 \pi d_{0} f_{c}}{c}\right)$ is the reference loss at reference distance $d_{0}$ following free-space path loss, the quantity $\alpha$ represents the path-loss exponent, $f_{c}$ is the carrier frequency, and $c$ is the speed of light.

\section{BEAM-CENTRIC HANDOVER}

TABLE I

LIST OF THE NOTATIONS

\begin{tabular}{l|l}
\hline$\lambda$ & Homogeneous PPP network density \\
$A$ & Bounded set in $\mathbb{R}^{2}$ \\
$n$ & Average number of BSs in area $A$ \\
$A_{b}$ & Area of a beam \\
$R$ & Beam radius at SNR threshold $\Gamma$ \\
$V\left(r, \theta_{v}\right)$ & Vehicle's location in polar coordinates \\
$\overrightarrow{\mathrm{BV}}$ & Line from a beam centre $B$ to a vehicle location $V$ \\
$\phi$ & Vehicle's moving direction. \\
$\psi$ & Vehicle's moving direction with respect to the Line $\overrightarrow{\mathrm{BV}}$ \\
$L$ & r.v. describing the beam displacement, $[0, R]$ \\
$k$ & r.v. describing the number of visible beams by \\
& a vehicle at the time of a handover event
\end{tabular}

\section{A. Scenario and Problem Formulation}

In this section, we first derive the vehicle displacement within a given beam, and then we extend our derivation to determine the longest vehicle displacement when multiple beams are visible by a vehicle. An example of the scenario is given in Fig. 1.

Without loss of generality, we consider a scenario setup where the mmWave BS deployment with an area $A$ follows a homogeneous PPP. Let $\Phi$ be a homogeneous PPP with mean $\lambda|A|$, and $\Phi(A)$ is the random variable (r.v.) describing the number of BSs in a bounded set $A \subset \mathbb{R}^{2}$, then the cumulative 
distribution function (CDF) of the number of BSs in $A$ for this PPP can be expressed as

$$
\mathbb{P}(\Phi(A) \leq n)=\sum_{k=0}^{n} \frac{(\lambda|A|)^{k}}{k !} \exp (-\lambda|A|) .
$$

Let $L$ be a r.v. describing a vehicle displacement within a beam, and $\mathbb{P}(L \leq l)$ be its $C D F$. At a particular handover event, a vehicle may detect $n$ BSs, hence $n$ beams ${ }^{1}$. Let $L_{i}$ each be the r.v. of the vehicle displacement of $i$-th beam, $i=1,2, \ldots, n$. Since each $L_{i}$ is independent and identically distributed (i.i.d.), the CDF of the maximum of the sample from the $n$ beams, denoted by $F_{L_{(n)}}(l)$, can be determined by applying results directly from Order Statistic, that is

$$
F_{L_{(n)}}(l)=[\mathbb{P}(L \leq l)]^{n} .
$$

The above result gives the CDF of the maximum vehicle displacement with $n$ available beams. Since the number of beams available to a vehicle at the time of a handover event is also a r.v., and its CDF is given in (4), by unconditioning $n$, we obtain the CDF of the maximum vehicle displacement for our scenario setup to be

$$
F_{\mathbf{L}_{\max }}(l)=\sum_{\mathbf{k}=0}^{\mathbf{n}} \frac{(\lambda|A|)^{k}}{k !} \exp (-\lambda|A|)[\mathbb{P}(L \leq l)]^{k}
$$

In the next subsection, we shall derive the vehicle displacement $\mathrm{CDF}$ for a given beam, i.e. $\mathbb{P}(L \leq l)$.

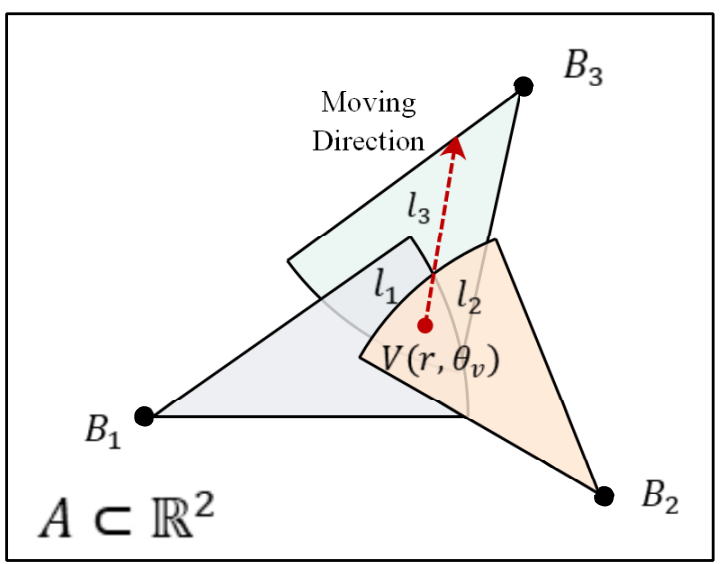

Fig. 1. Beam decision scenario when vehicle falls under the coverage of multiple beams. In the figure, $l_{1}, l_{2}$ and $l_{3}$ represent the vehicle displacements within the coverage of beams $B_{1}, B_{2}$ and $B_{3}$ respectively in the case that the vehicle decides to connect any of them at the decision point $V\left(r, \theta_{v}\right)$. In this example, the vehicle should choose $B_{3}$ for handover since it offers the vehicle the longest displacement. Note that all beams share identical configuration of beam width $\left(\theta_{s}\right)$ and coverage range (determined by pre-defined SNR threshold $\Gamma$ ).

\section{B. Analysis of Vehicle Displacement within a Beam}

A handover event is triggered when a vehicle moves outside of the coverage of its current beam connection. Since the location of any beam follows PPP, a handover event may

\footnotetext{
${ }^{1}$ User can only use one beam per BS at a given time, so the numbers of available BSs and beams are identical.
}

occur at any random location uniformly distributed in $A$. At this location, the vehicle may detect a number of beams from its nearby BSs for the handover, and its location within each beam is also random with uniform distribution. We assume that the vehicle continues to maintain the same direction moving constantly after the handover. Given the directional antenna feature of mmWave BS, the considered beam coverage is assumed to have a shape of a sector.

We consider a beam $b$ defined by its direction $\theta_{b}$, beam width angle $\theta_{s}$ and radius $R$, with a coverage area $A_{b}$. At the time of handover decision, the beam is visible by a vehicle $v$ at a location with polar coordinate $V\left(r, \theta_{v}\right)$ moving in a particular direction. Assuming that the vehicle has handed over to this beam and it continues to move in the same direction at $\phi$, the vehicle displacement within this beam can be determined by the distance between the current location of the vehicle and a point at the edge of the beam where the vehicle departs some time later. The probability that the vehicle displacement is shorter than a particular value $l$ can be determined by

$$
\mathbb{P}(L \leq l)=\frac{1}{A_{b}} \int_{0}^{R} \int_{\Theta_{1}}^{\Theta_{2}} \int_{0}^{\Phi} \frac{I_{L}\left(\hat{l}_{r, \theta_{v}, \phi}\right)}{2 \pi} R d \phi d \theta d r
$$

where $\Phi=2 \pi, \Theta_{1}=\theta_{b}-\frac{\theta_{s}}{2}$ and $\Theta_{2}=\theta_{b}+\frac{\theta_{s}}{2}$. Note that $I_{L}\left(\hat{l}_{r, \theta_{v}, \phi}\right)$ is an indicator function defined as

$$
I_{L}\left(\hat{l}_{r, \theta_{v}, \phi}\right)= \begin{cases}1 & \text { if } \hat{l}_{r, \theta_{v}, \phi} \leq l \\ 0 & \text { otherwise }\end{cases}
$$

To determine whether the condition $\hat{l}_{r, \theta_{v}, \phi} \leq l$ is satisfied, we need to calculate $\hat{l}_{r, \theta_{v}, \phi}$, which is the distance between the vehicle $V$ located at $\left(r, \theta_{v}\right)$ and the edge of the beam where the vehicle leaves the beam, as illustrated in Fig. 2.

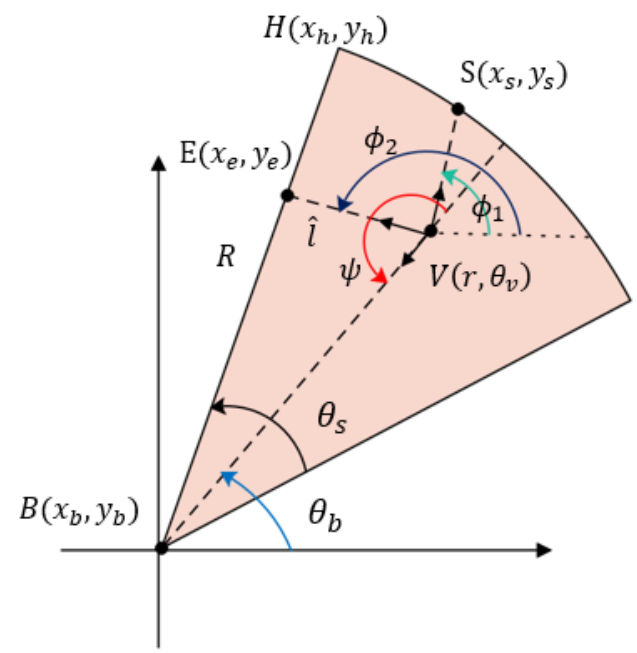

Fig. 2. An example of vehicle displacement within single beam of mmWave BS. $\phi_{1}$ and $\phi_{1}$ represent the vehicle's two different moving directions when it leaves the beam coverage through the points $S\left(x_{s}, y_{s}\right)$ and $E\left(x_{e}, y_{e}\right)$, respectively. 
The calculation will involve finding the intersection points on the radii and arc of the beam where the vehicle leaves.

For the intersection point on the radius, say Point $E$ at Cartesian coordinate of $\left(x_{e}, y_{e}\right)$ in Fig. 2, can be determined by finding the intersection of the line equation for beam radius, $\ell_{H}$, with slope $m_{h}$, and the line equation of the vehicle trajectory, $\ell_{V}$, with slope $m_{v}$ as follows

$$
\left\{\begin{array}{l}
\ell_{H}: y=m_{h}\left(x-x_{b}\right)+y_{b} \\
\ell_{V}: y=m_{v}\left(x-x_{v}\right)+y_{v}
\end{array}\right.
$$

where the distance between Point $E$ and $V$ can be determined. The same calculate applies for intersection point on the other side of the radius.

To find the point where vehicle leaves the arc section, say Point $S$ at Cartesian coordinate of $\left(x_{s}, y_{s}\right)$ in Fig. 2, we simply need to find the intersection point between $\ell_{V}$ and the equation of the circle, $c_{B}$ as follows.

$$
c_{B}: R^{2}=\left(x-x_{b}\right)^{2}+\left(y-y_{b}\right)^{2} .
$$

Having obtained Point $S$, we can simply calculate the distance between Points $S$ and $V$.

\section{Fuzzy Logic (FL) Based Handover Decision}

FL has been widely used in literature for designing decision making algorithms [11]. FL follows human reasoning in deducting meaningful data from multiple conflicting parameters when uncertainty and imprecision is present. Since it is unknown where vehicle will head on the beam boundary, in this paper, we take advantage of this fuzziness and employ FL to deduce the vehicle displacement value given a beam. We implement FL-based distributed handover decision algorithm by considering vehicle location and direction of travel when it falls under coverage of multiple beams. Here we utilise fuzzy system to roughly estimate the vehicle moving displacement in a candidate beam. As shown in Fig. 3 in our case fuzzy system takes three inputs and produce single output which gives a clue on the displacement value under each candidate beam. Then the vehicle ranks the FL outcome and decides which beam that is likely to provide the longest displacement.

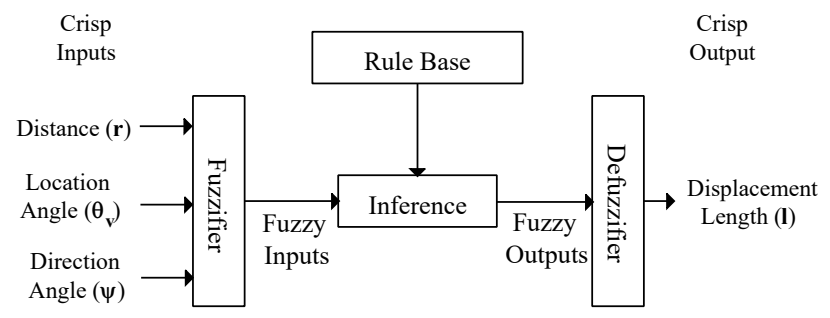

Fig. 3. Fuzzy Logic design.

The fuzzy rule base defined in our design consists of $3 \times 3 \times 6=54^{2}$ for the proposed Fuzzy System (FS). In the proposed FS, vehicle location parameters $r$ and $\theta_{v}$ and moving

\footnotetext{
${ }^{2}$ Note that we only shows rules which result in high $L$ outcome values in Table II as we are more interested in the beams which can provide higher displacement.
}

TABLE II

FUZZY RULES

\begin{tabular}{ccccc}
\hline \multirow{2}{*}{ No } & \multicolumn{3}{c}{ IF } & THEN \\
\cline { 2 - 5 } & r & $\theta_{v}$ & $\psi$ & $L$ \\
\hline 1 & Near & Low & Very Low & High \\
2 & Near & Medium & Very Low & High \\
3 & Near & Medium & Very High & High \\
4 & Near & High & Very High & High \\
5 & Far & Low & Low Medium & High \\
6 & Far & Medium & Low Medium & High \\
7 & Far & Medium & High Medium & High \\
8 & Far & High & High Medium & High \\
\hline
\end{tabular}

direction angle $\psi$ which are in the range $[0, R],\left[\Theta_{1}, \Theta_{2}\right]$ and $[0,2 \pi]$, respectively, represent input variables to produce output variable $l$ which is in the range $[0, R]$.

The fuzzy rules is presented in Table II showing the IFTHEN rule used in the proposed FS for the outcome where $L$ is deemed high, and other cases not shown in the table produce an outcome where $L$ is deemed low. For example, if $r$ is near, $\theta_{v}$ is medium and $\psi$ is very low, $L$ is deemed high. This abides by the case when vehicle at location $V\left(r, \theta_{v}\right)$ is moving towards the beam centre $B\left(x_{b}, y_{b}\right)$ with angle $\psi$ in respect of $\overrightarrow{\mathrm{BV}}$ as shown in Fig. 2. Note that even smaller difference in angle $\psi$ can make significant impact on output $L$ due to beam shape enclosed by one arc and two radii. Hence, this impact can be higher when vehicle is closer to beam centre and moving in the perpendicular direction to the line $\overrightarrow{\mathrm{BV}}$. For this reason we consider six fuzzy sets (very low, low, low medium, high medium, high, very high) for angle $\psi$ to capture small changes while considering three fuzzy sets for $r$ (i.e., near, medium and far) and $\theta_{v}$ (i.e.,low, medium and high). Here, fuzzy sets are modelled with triangular membership function which designates a degree of membership in the range $[0,1]$ to each fuzzy set.

\section{Performance Evaluation}

In this work, average throughput and handover cost are used to evaluate beam-centric handover performance in dense mmWave networks. Firstly, since inter-cell interference is neglected here, signal-to-noise ratio (SNR) in $\mathrm{dB}$ is calculated as

$$
S N R=P_{r}-P_{N}
$$

where noise power $P_{N}$ is

$$
P_{N}=10 \cdot \log _{10}(W)+N_{f}+N F
$$

where $W$ is transmission bandwidth and $N_{f}$ and $N F$ are noise floor value and noise figure respectively.

By using (11), the average throughput $(A T)$, i.e. bit/sec., before considering handover cost can be expressed as

$$
A T=W \times \log _{2}(1+S N R) .
$$

In order to evaluate handover performance, simulation based inter-cell and inter-beam handover rate are used to calculate 
the handover cost. Note that since the ICH event involves two different mmWave BSs, it incurs higher signalling overhead than IBH which occurs between two different beams of the same mmWave BS. Let $\left(H O R_{1}, d_{1}\right)$ and $\left(H O R_{2}, d_{2}\right)$ be a pair of handover rate and handover delay for $\mathrm{ICH}$ and $\mathrm{IBH}$, respectively. Note that the handover rates are expressed as the average number of handovers per second, i.e., total number of handovers normalised by simulation time. Hence, the total handover cost $D_{H O}$, i.e. handover delay per unit time, can be calculated as

$$
D_{H O}=H O R_{1} \times d_{1}+H O R_{2} \times d_{2} .
$$

Now when we include handover cost $D_{H O}$ and control overhead $u$ which is in the range $(0,1)$, the average throughput $(A T)$ in (13) becomes

$$
A T \times(1-u) \times\left(1-\min \left(1, D_{H O}\right)\right) .
$$

In this study, we note that all evaluated schemes are based on that once the vehicle performed handover to certain beam it will continue to stay under the coverage of the same beam unless SNR drops below the threshold $\Gamma=5.7 \mathrm{~dB}^{3}$

\section{A. Simulation And Numerical Results}

In this section we provide simulation and theoretical results to validate the developed analytical models for vehicle displacement within a beam and theoretical upper-bound vehicle displacement among all visible beams to the vehicle. We then evaluate the performance of proposed FL-based distributed algorithm in terms of handover cost and throughput under different handover delay assumptions and varying vehicle velocities. Simulation parameters are summarised in Table III.

TABLE III

SimUlation PARAMETERS

\begin{tabular}{ll}
\hline Parameter & Value \\
\hline Number of beams per mmBS $\left(N_{b}\right)$ & 20 \\
Carrier Frequency $\left(f_{c}\right) /$ Bandwidth $(W)$ & $28 \mathrm{GHz} / 500 \mathrm{MHz}$ \\
Transmit power $\left(P_{t}\right)$ & $30 \mathrm{dBm}$ \\
Path loss exponent $(\alpha)^{4}$ & 3 \\
Angle of sector $\left(\theta_{s}\right)$ & $18^{\circ}$ \\
Noise floor value $\left(N_{f}\right)$ & $-174 \mathrm{dBm}$ \\
Noise figure $(N F)$ & $6 \mathrm{~dB}$ \\
Simulation time & $100 \mathrm{~s}$ \\
Sampling time slot $\left(T_{s}\right)$ & $0.1 \mathrm{sec}$. \\
Simulation area & $1000 \mathrm{~m} \mathrm{x} 1000 \mathrm{~m}$ \\
\hline
\end{tabular}

In Fig. 4, CDF results for a vehicle displacement within single beam are given to validate both simulation and analytical model. Result for analytical model is based on displacement distribution $\mathbb{P}(L \leq l)$ derived in (7). Results show that nearly $90 \%$ of displacements are less than $40 \mathrm{~m}$ which is quite small as compared to maximum displacement $R=135 \mathrm{~m}$.

${ }^{3} \mathrm{SNR}$ shall be greater than about $5 \mathrm{~dB}$ to achieve the target BLock Error Rate (BLER), i.e. $10^{-} 6$, required by successful URLLC packet transmission [12].

${ }^{4}$ Typical omnidirectional path loss exponent is considered [13].
Fig. 5 shows the CDF of the vehicle displacement for different beam based handover decision schemes. In the result, Max-Displacement is simulation results where a vehicle preruns the simulation by exhausting all possibilities of the beams, and then runs the simulation by picking the beam with maximum displacement based on the pre-run data. The purpose of obtaining this result is to determine the upperbound simulation results. We also compare the result with the analytical upper-bound solution from (6). It is shown that good agreement has been achieved showing accuracy of our analytical model.

We can also see from Fig. 5 that our proposed FL-based solution gives reasonably good performance. Compared with Best-SNR solution always selecting the beam to offer the highest SNR, FL-based solution shows far superior performance as it records closer performance to the theoretical upper-bound result. It achieves this by specifically avoiding very short lengths of displacement, and this helps avoiding any unneeded or ping-pong handovers. In the Best-SNR solution, almost $90 \%$ of lengths are less than $25 \mathrm{~m}$ where it is less than $20 \%$ in FLBased solution and nearly $5 \%$ for the upper-bound results.

Fig. 6 shows the handover cost with two different handover delays and varying velocity values. As expected, handover cost increases with increasing velocity values due to rise in the number of the handovers per second. $\mathrm{HO}$ cost for Best-SNR is doubled of FL-based at speed $10 \mathrm{~m} / \mathrm{s}$ and the gap becomes wider with higher speed. One can also observe that FL-based solution in terms of handover cost and are close to Max-Displacement. As handover delays for $\mathrm{ICH}$ and IBH $\left(d_{1}, d_{2}\right)$ is doubled from $(0.35,0.175)$ to $(0.7,0.35)$ [8], the handover cost becomes much more severe for Best-SNR solution compared to the others.

In Fig. 7, we compare the throughput performance taking handover cost into account. As expected, throughput performance degrades for all cases as velocity increases due to increasing handover cost. Interestingly, FL-based solution appears lower on throughput, but under high mobility and handover delay, its performance matches that of MaxDisplacement solution. It is clear that the FL-based solution

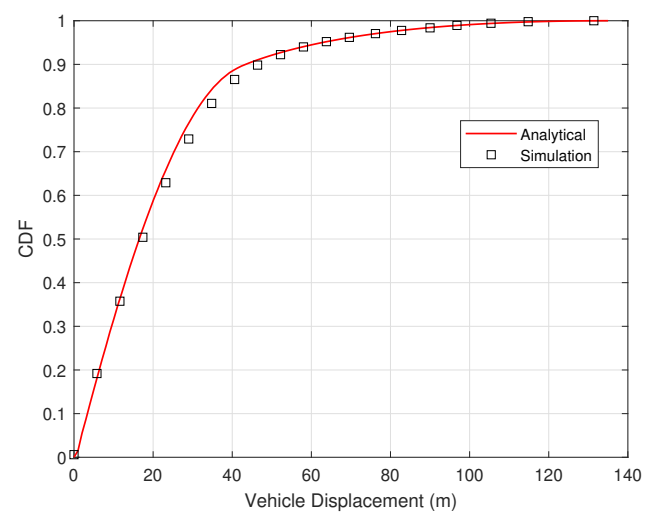

Fig. 4. CDF comparison between simulation and analytical based vehicle displacement within a single beam with radius $R=135 \mathrm{~m}$. 


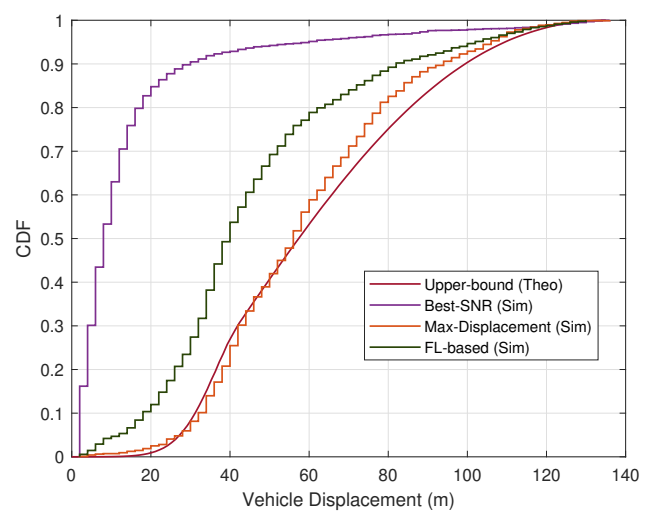

Fig. 5. Cumulative distribution of vehicle displacement among the selected beams for different solutions when $\lambda=\frac{1}{40^{2} \pi} \mathrm{m}^{-2}$.

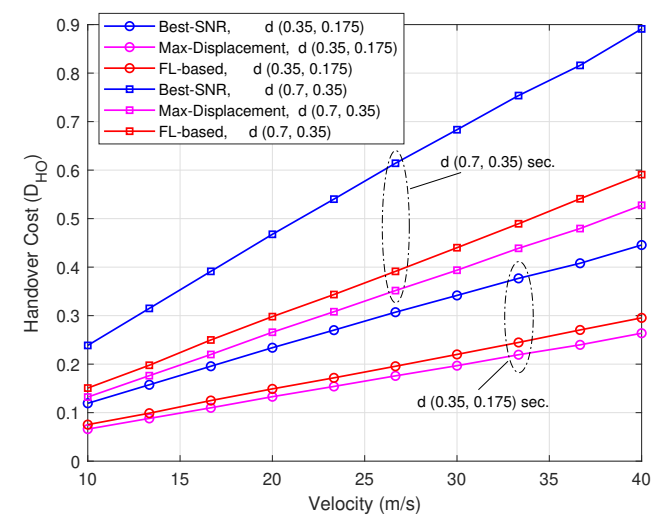

Fig. 6. Handover cost versus velocity for $\lambda=\frac{1}{80^{2} \pi} m^{-2}$.

is less sensitive to mobility in dense networks since it can accurately identify a longer connectivity in the first place.

\section{CONCLUSIONS}

In this study, we analytically derived the vehicle displacement distribution in a beam coverage. We then formulated an upper-bound limit for vehicle displacement distribution based on available number of beams. Moreover, we designed the FL based beam-centric distributed handover decision algorithm to improve vehicle displacement. Simulation results show the FL based longest vehicle displacement decision mechanism is superior to the Best-SNR based approach in terms of handover cost and overall throughput. The Best-SNR approach could be a good option at low speed but it becomes less favourable with increase of vehicle's speed or handover delay. In future work, we will extend this work for the multi-vehicle scenario where the longest beam might not always be available to each user.

\section{ACKNOWLEDGMENT}

This work was supported by Jaguar Land Rover and the UK-EPSRC grant EP/N01300X/1 as part of the jointly funded Towards Autonomy: Smart and Connected Control (TASCC) Programme. We also would like to acknowledge the support

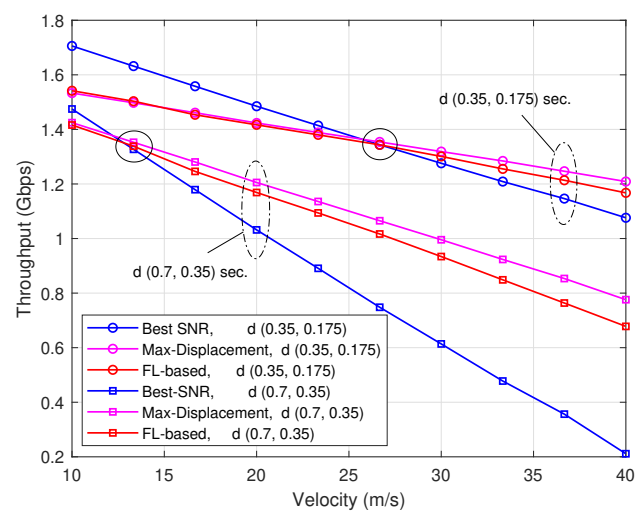

Fig. 7. Average throughput versus velocity for $\lambda=\frac{1}{80^{2} \pi}$.

of the 5GIC members for this work. The first author is funded by the Republic of Turkey Ministry of National Education. The third author is funded by the EU H2020 research and innovation programme 5G-HEART project under grant 857034 .

\section{REFERENCES}

[1] Y.-J. Chen, T. Hsu, and L.-C. Wang, "Improving handover performance in 5G mm-Wave HetNets," in Proc. IEEE Global Communications Conference (GLOBECOM), 2017, pp. 1-6.

[2] Y. Niu, Y. Li, D. Jin, L. Su, and A. V. Vasilakos, "A survey of millimeter wave communications (mmWave) for 5G: opportunities and challenges," Wireless networks, vol. 21, no. 8, pp. 2657-2676, 2015.

[3] J. Stańczak, "Mobility Enhancements to Reduce Service Interruption Time for LTE and 5G," in Proc IEEE Conference on Standards for Communications and Networking (CSCN). IEEE, 2016, pp. 1-5.

[4] R. Arshad, H. ElSawy, S. Sorour, T. Y. Al-Naffouri, and M.-S. Alouini, "Velocity-aware handover management in two-tier cellular networks," IEEE Trans. Wireless Commun., vol. 16, no. 3, pp. 1851-1867, 2017.

[5] — "Handover management in dense cellular networks: A stochastic geometry approach," in Proc. IEEE Int'l Conference on Communications (ICC), 2016, pp. 1-7.

[6] E. Demarchou, C. Psomas, and I. Krikidis, "Mobility management in ultra-dense networks: Handover skipping techniques," IEEE Access, vol. 6, pp. 11921-11930, 2018.

[7] B. Li, H. Zhang, P. Hao, and J. Li, "Sojourn time estimation-based small cell selection in ultra-dense networks," in Proc. IEEE 28th Annual International Symposium on Personal, Indoor, and Mobile Radio Communications (PIMRC), 2017, pp. 1-5.

[8] Z. Li and W. Wang, "Handover Performance in Dense MmWave Cellular Networks," in Proc. IEEE 10th International Conference on Wireless Communications and Signal Processing (WCSP), 2018, pp. 1-7.

[9] J. S. Kim, W. J. Lee, and M. Y. Chung, "A multiple beam management scheme on $5 \mathrm{G}$ mobile communication systems for supporting high mobility," in Proc. IEEE Int'l Conference on Information Networking (ICOIN), 2016, pp. 260-264.

[10] C. A. Balanis, Antenna theory: analysis and design. John Wiley \& Sons, 2016.

[11] R. M. Al-Kiyumi, C. H. Foh, S. Vural, P. Chatzimisios, and R. Tafazolli, "Fuzzy logic-based routing algorithm for lifetime enhancement in heterogeneous wireless sensor networks," IEEE Trans. Green Commun. Netw., vol. 2, no. 2, pp. 517-532, 2018.

[12] M. Sybis, K. Wesolowski, K. Jayasinghe, V. Venkatasubramanian, and V. Vukadinovic, "Channel coding for ultra-reliable low-latency communication in $5 \mathrm{~g}$ systems," in 2016 IEEE 84th vehicular technology conference (VTC-Fall), 2016, pp. 1-5.

[13] T. S. Rappaport, G. R. MacCartney, M. K. Samimi, and S. Sun, "Wideband millimeter-wave propagation measurements and channel models for future wireless communication system design," IEEE transactions on Communications, vol. 63, no. 9, pp. 3029-3056, 2015. 